DFTT-46/95

hep-lat/9510019

\title{
Width of Long Colour Flux Tubes in Lattice Gauge Systems
}

\author{
M. Caselle ${ }^{a}$, F. Gliozzi ${ }^{a}$, U. Magnea ${ }^{a, b}$ and S. Vinti ${ }^{a}$ \\ ${ }^{a}$ Dipartimento di Fisica Teorica dell'Università di Torino \\ Istituto Nazionale di Fisica Nucleare, Sezione di Torino \\ Via P.Giuria 1, I-10125 Torino, Italy 用 \\ ${ }^{b}$ State University of N.Y. at Stony Brook, Stony Brook, NY 11794 USA
}

\begin{abstract}
In the confining phase of any gauge system the mean squared width of the colour flux tube joining a pair of quarks should grow logarithmically as a function of their distance, according to the effective string description of its infrared properties. New data on $3 D \mathbb{Z}_{2}$ gauge theory, combined with high precision data on the interface physics of the $3 D$ Ising model fit nicely this behaviour over a range of more than two orders of magnitude.
\end{abstract}

*e-mail: caselle, gliozzi, vinti @to.infn.it

${ }^{\dagger} \mathrm{e}-$ mail: blom @to.infn.it 


\section{Introduction}

The mechanism of confinement for abelian and non-abelian gauge theories in three or four space-time dimensions seems well described by the old conjecture [1] that the vacuum behaves as a dual superconductor. The dual Meissner effect squeezes the colour field generated by a pair of quark sources into a thin flux tube (the dual version of the Abrikosov vortex), generating a static potential proportional to the interquark distance. Numerical studies of the distribution of the colour flux around the quark sources [2, 3, 4 seem to support this picture.

According to another old conjecture [6, 7, 8], this flux tube can vibrate as a free string. There are many interesting and impressive quantum effects associated to these string vibrations. One of them seems to conflict with a property of the dual Abrikosov vortices: the intrinsic thickness of these is a physical quantity which is independent of the interquark distance and is fixed by the parameters of the Ginzburg-Landau formulation of the superconductivity, while in the string picture the width of the flux tube grows logarithmically as a function of the interquark distance [8].

In this paper we will show compelling numerical evidence of this logarithmic growth. However this does not mean that the picture of the dual Abrikosov vortex does not work. Simply, the way one defines the mean squared width of the flux tube in the lattice simulation also takes into account the quantum effect of the vibration modes of the dual Abrikosov vortex, yielding an effective width which is larger than the intrinsic thickness of a non-vibrating vortex.

It is worthwhile to stress that the dual superconductor nature of the confining vacuum and the string-like behaviour of the colour flux tube are conceptually two independent properties of the theory. Indeed in the strong coupling regime, before the roughening transition, i.e. in the region where the strong coupling expansion of the string tension $\sigma$ converges, the dual Meissner effect is already operating and the colour flux tube connecting 
pairs of quark sources has a string-like form, however cannot vibrate. Hence in this strong coupling phase the dual Abrikosov vortices, like those of the ordinary superconducting media, do not exhibit any kind of quantum string effects.

At the roughening point, the colour flux tube of whatever $3 D$ or $4 D$ gauge theory undergoes a transition towards a rough phase (which is the one connected to the continuum limit), where it can vibrate freely and the energy stored in its vibration modes is no longer negligible. The collective degrees of freedom describing such a string-like motion can be described by a $2 D$ field theory known as effective string theory.

It is widely believed that the roughening transition is described by the universality class of Kosterlitz and Thouless [9]. Accordingly, the renormalization group equations show that in the rough phase the effective string theory describing the dynamics of the flux tube flows at large scales towards a massless free field theory. Thus, for large enough interquark separations, it is not necessary to know explicitly the specific form of the effective string action describing the behaviour of the flux tube, but only its infrared massless limit. This simple, general fact has some important implications because the limit action is critical in the whole rough phase. As a consequence, it produces universal (i.e. gauge group independent) finite size effects. Moreover, because this theory is also infrared divergent, such finite size effects are expected to be rather strong.

The effects we shall describe require highly accurate numerical data that at present can hardly be reached in $4 \mathrm{D} S U(2)$ and $S U(3)$ gauge models.

Luckily, these effects can be checked accurately in the $3 D \mathbb{Z}_{2}$ gauge model which is the simplest prototype of gauge theory.

\section{Width of the Colour Flux Tube}

The logarithmic behaviour of the mean squared width $w^{2}(R)$ of the colour flux tube as a function of the interquark distance $R$ has been predicted many years ago by Lüscher, Münster and Weisz [8] in the framework of the effective string picture of gauge systems. 
We shall rederive and refine this universal law by directly using some exact results on the two dimensional free gaussian model in a finite box and compare it with Monte Carlo simulations on the $3 D \mathbb{Z}_{2}$ gauge model.

We shall see that the free gaussian model describes quite accurately this phenomenon.

\subsection{Results from the Gaussian Limit}

In order to fix notations, let us recall that the simplest way to implement the effective string picture of the colour flux tube is to assume that the vacuum expectation value of a large Wilson loop $W(C)$ can be represented by the functional integral

$$
\langle W(C)\rangle=\int \prod_{i=1}^{D-2}\left[D h_{i}\right] \mathrm{e}^{-\int \mathrm{d}^{2} \xi \mathcal{L}\left(h_{i}\right)} .
$$

For simplicity $C$ is taken planar and $\xi^{\alpha}, \alpha=1,2$ are the coordinates of the planar domain $\mathcal{D}$ bounded by $C . h_{i}\left(\xi^{1}, \xi^{2}\right)$ are the transverse coordinates of the string describing the configurations of the flux tube in its space-time evolution. They are the fields entering in the effective string action $S=\int \mathrm{d}^{2} \xi \mathcal{L}\left(h_{i}\right)$, which is largely unknown, but is believed to flow to the free gaussian model for very large loops $C$, as explained in the introduction. More precisely we have

$$
S \rightarrow \sigma A+\int_{\mathcal{D}} \mathrm{d}^{2} \xi \sum_{i=1}^{D-2} \frac{\sigma}{2}\left(\partial_{i} h_{i}\right)^{2}
$$

where $\sigma$ is the string tension and $A=\int_{\mathcal{D}} d^{2} \xi$ is the area of the planar domain. Eq. (2) can be considered as the first two terms of an expansion in the adimensional parameter $1 / \sigma A$. Recently, the first non-gaussian correction of such an expansion has also been studied [10, 11].

The mean squared width of the flux tube generated by this Wilson loop is defined as the sum of the mean square deviations of the transverse coordinates $h_{i}\left(\xi^{1}, \xi^{2}\right)$ of the underlying string, i.e.

$$
w^{2}\left(\xi^{1}, \xi^{2}\right)=\sum_{i=1}^{D-2}\left\langle\left(h_{i}\left(\xi^{1}, \xi^{2}\right)-h_{i}^{C M}\right)^{2}\right\rangle
$$


where $h_{i}^{C M}$ is the transverse coordinate of the center of mass of the flux tube. In terms of the Green functions $G_{i}$ we get

$$
\sigma w^{2}(\xi)=\sum_{i=1}^{D-2}\left\{G_{i}(\xi, \xi+\varepsilon)-\frac{2}{A}\left[\int_{\mathcal{D}} d^{2} \xi^{\prime}\left(G_{i}\left(\xi, \xi^{\prime}\right)-\frac{1}{2 A} \int_{\mathcal{D}} d^{2} \xi^{\prime \prime} G_{i}\left(\xi^{\prime \prime}, \xi^{\prime}\right)\right)\right]\right\}
$$

where $\varepsilon$ is a UV cut-off, $\sigma$ is the string tension and the Green function is defined as

$$
G_{i}\left(\xi, \xi^{\prime}\right)=\sigma\left\langle h_{i}(\xi) h_{i}\left(\xi^{\prime}\right)\right\rangle
$$

The vacuum expectation value is taken with respect to the string action $S$. In the infrared limit the Green function fulfills the free field equation

$$
-\Delta G\left(\xi, \xi^{\prime}\right)=\delta^{(2)}\left(\xi-\xi^{\prime}\right)
$$

where for simplicity the index $i$ of the transverse direction has been omitted. Instead of considering the usual expansions of $G$ in terms of the eigenfunctions of $\Delta$ it is possible (and much more useful) to write it in a more compact form.

Indeed the problem of finding the Green function for an arbitrary, simply connected region $\mathcal{D}$ can be solved in a closed form once a conformal mapping $z=\xi^{1}+i \xi^{2} \rightarrow \ell$ of $\mathcal{D}$ onto the unit circle $|\ell|=1$ is found which maps $z^{\prime}=\xi^{\prime 1}+i \xi^{\prime 2}$ into the origin $\ell=0$. Denoting by $\ell_{z^{\prime}}(z)$ the analytic function providing us with such a mapping, it is immediate to verify that the real function $f_{z^{\prime}}(z, \mathcal{D})=\log \left|\ell_{z^{\prime}}(z)\right|$ is harmonic in the punctured set $\mathcal{D} \backslash\left\{z^{\prime}\right\}$ (hence $\Delta f=0$ for $z \neq z^{\prime}$ ), vanishes at the boundary $\partial \mathcal{D}$ and diverges $\operatorname{logarithmically}$ as $\log \left|z-z^{\prime}\right|$ for $z \rightarrow z^{\prime}$. It follows that the Green function is given by

$$
G_{\mathcal{D}}\left(z, z^{\prime}\right)=-\frac{1}{2 \pi} f_{z^{\prime}}(z, \mathcal{D})
$$

Some explicit examples can be found in Tab. I and will be discussed later.

Inserting such expressions in Eq.(近), we can evaluate analytically the shape of the flux tube as a function of the coordinate $z$ inside the domain $\mathcal{D}$. One can observe a very steep increase of $w^{2}(z)$ at the boundary of $\mathcal{D}$ followed by a plateau covering approximately the 
$90 \%$ of the domain, where $w^{2}(z)$ grows very slowly an reaches its maximum in the center $z_{o}$ of the domain. For instance, in the case of an infinite strip of width $R$ we get

$$
2 \pi \sigma w^{2}(r)=\log \frac{\left|\sin \left(2 r+\frac{R_{c}}{R}\right)\right|}{\left|\sin \left(\frac{R_{c}}{R}\right)\right|},
$$

where $r=\frac{\pi}{2 R} x, 0 \leq x \leq R$, and the UV cut-off has been reabsorbed in the length scale $R_{c}=\frac{\pi \varepsilon}{2}$. As a measure of the total width of the flux tube it is often used in the literature the integrated quantity

$$
w^{2}=\frac{1}{A} \int_{\mathcal{D}} d^{2} \xi w^{2}\left(\xi^{1}, \xi^{2}\right)
$$

However in the applications to the gauge models this quantity may be affected by nonuniversal corrections due to the self-interactions of the quark line at the boundary. For this reason we prefer to use instead the value of the mean squared width measured at the symmetry point of the domain:

$$
w_{o}^{2}=w^{2}\left(\xi_{o}^{1}, \xi_{o}^{2}\right)
$$

For instance, in the Wilson loop of size $R \times T$ we have obviously $\xi_{o}^{1}=\frac{R}{2}$ and $\xi_{o}^{2}=\frac{T}{2}$.

Tab. I

\begin{tabular}{cccc}
\hline $\mathcal{D}$ & b.c. & Green function & $2 \pi \sigma \Delta w^{2}$ \\
\hline Disk & fixed & $G_{D}\left(z, z^{\prime}\right)=-\frac{1}{2 \pi} \log \left|R \frac{z-z^{\prime}}{R^{2}-z \bar{z}^{\prime}}\right|$ & 0.8105 \\
Square & fixed & $G_{R}\left(z, z^{\prime}\right)=-\frac{1}{2 \pi} \log \left|\frac{\sigma\left(z-z^{\prime}\right) \sigma\left(z+z^{\prime}\right)}{\sigma\left(z-\bar{z}^{\prime}\right) \sigma\left(z+\bar{z}^{\prime}\right)}\right|$ & -0.0139 \\
Strip & fixed & $G_{S}\left(z, z^{\prime}\right)=-\frac{1}{2 \pi} \log \left|\frac{\sin \left(\pi\left(z-z^{\prime}\right) / 2 R\right)}{\sin \left(\pi\left(z+\bar{z}^{\prime}\right) / 2 R\right)}\right|$ & 0.8587 \\
Torus & periodic & $\mathcal{G}\left(z-z^{\prime}\right)$ & 0 \\
\hline
\end{tabular}

The closed form of the Green functions for some simple domains is given together with the difference between the mean squared width as defined in Eq.s (4.10) and that relative to a square torus. 
For our purposes, the relevant property of the conformal mapping $\ell$ is that one can perform an arbitrary scale transformation $z \rightarrow \Lambda z$ without destroying the conformal character of $\ell$. More precisely we can write

$$
f_{z^{\prime}}(z, \mathcal{D})=f_{\Lambda z^{\prime}}\left(\Lambda z, \mathcal{D}_{\Lambda}\right)
$$

where $\mathcal{D}_{\Lambda}$ denotes the scaled domain. A direct consequence is that it is always possible to fix the area of the scaled domain $\mathcal{D}_{\Lambda}$ to an arbitrary value, say 1 , without changing the Green function. It follows that the integration of the finite part $G\left(z, z^{\prime}\right)$ in Eq. (4) cannot depend on the size of the domain $\mathcal{D}$ but only on its shape. On the contrary the UV divergent part gives a contribution which grows logarithmically with the size of the domain. This can be simply understood as follows: if $R$ is a typical linear dimension of the domain $\mathcal{D}$, the scaling property described above implies that the cut-off should appear always in the ratio $\varepsilon / R$. On the other hand, for $\varepsilon<<R$ the Green function behaves like $-\frac{1}{2 \pi} \log (\varepsilon)$, yielding the logarithmic law

$$
w_{o}^{2}=\frac{1}{2 \pi \sigma} \log \left(R / R_{c}\right)
$$

where the UV cut-off has been absorbed in the definition of the scale $R_{c}$.

Note that the above equation is meaningful only for $R>R_{c}$. This inequality has a simple physical meaning: the dynamics of the flux tube is described by a truly free field theory only at large distances; the cut-off $\varepsilon$ sets up the ultraviolet scale $R_{c}$ below which either the free-field approximation breaks down or the internal degrees of freedom of the flux tube become important. Thus $R_{c}$ gives us a very rough estimate of the intrinsic thickness of the flux tube.

The absolute value of $R_{c}$ is not directly calculable, being proportional to the UV cutoff. We can however evaluate the ratios among the $R_{c}$ 's of different domains making the very mild assumption that the UV cut-off $\varepsilon$ cannot depend on the size and the shape of the $\mathcal{D}$ domain (which are on the contrary infrared properties). For later convenience, we shall use as reference scale $R_{p}$ associated to a squared box with periodic boundary 
conditions (i.e. a torus). Also in this case the the Green function $\mathcal{G}$ can be expressed in a closed form. More generally, for a torus of sides $R \times T$ we have (see for instance Ref. [1] ):

$$
\mathcal{G}(z)=-\frac{1}{2 \pi} \ln |\sigma(z)|+\frac{\pi E_{2}(i t)}{12 R^{2}} \Re e\left(z^{2}\right)+\frac{1}{2 R T}(\Im m z)^{2},
$$

where $\sigma(z)$ is the Weierstrass sigma function, defined through the infinite product

$$
\sigma(z)=z \prod_{\omega \neq 0}\left(1-\frac{z}{\omega}\right) \mathrm{e}^{z / \omega+\frac{1}{2}(z / \omega)^{2}}, \quad \omega=m R+i n T .
$$

The set $\{\omega\}$ is known as the period lattice and $E_{2}(i t)$ is the first Eisenstein series with $t=T / R$. For more details see also the Appendix.

Denoting with $w_{p}^{2}(R)=\frac{1}{2 \pi \sigma} \log \left(R / R_{p}\right)$ the mean squared width of the flux tube generated by taking $\mathcal{D}$ to be the square box of reference, it follows that the difference $\Delta w^{2}(R)=w_{o}^{2}(R)-w_{p}^{2}(R)$ does not depend on $R$ but is a calculable function of the shape of $\mathcal{D}$.

In Tab. I one can find the closed form of the Green function and the corresponding $\Delta w^{2}$ of some simple domains like a disk of radius $R$, a square of side $R$, an infinite strip of width $R$ and the reference torus of size $R \times R(R=T)$. It is important to notice that the Weierstrass sigma function entering the definition of the Green function of the square of side $R$ corresponds to a torus of double size. For a rectangle of size $R \times T$ the Green function has the same formal expression as the square in terms of $\sigma(z)$, which now is defined on a torus of size $2 R \times 2 T$.

The detailed calculation of $w^{2}$ for the torus (which is interesting in the physics of fluid interfaces) and for the rectangular Wilson loop can be found in the Appendix. The result is drawn in Fig. 1, where the behaviour of $\Delta w^{2}$ for a rectangle as a function of the aspect ratio $t=T / R$ (dashed line) is compared with that of a rectangular torus with the same shape $t$ (continuous line) and with the value for the infinite strip (dotted line). Note that the almost linear increase of $\Delta w^{2}$ for the torus agrees with the fact that for large $t$ the theory becomes almost one-dimensional and describes the interface of a $2 D$ Ising model, where the mean squared width is known to grow linearly with the size. 
This behaviour of $\Delta w^{2}$ calculated in the free gaussian model as a function of the shape and of the boundary conditions (periodic and fixed) can be easily checked in the $2 D X Y$ model (in Fig. 1, open circles and triangles respectively) which is believed to belong to the Kosterlitz-Thouless universality class and certainly flows to the free gaussian model at large scales. The other numerical data reported in Fig. 1 (black dots) are data extracted from the $3 D \mathbb{Z}_{2}$ gauge model simulations which will be discussed in the next section.

If one is able to evaluate, besides the effective squared width, also the higher moments $w^{(2 n)}$, defined by

$$
w^{(2 n)}=\frac{1}{A} \sum_{i=1}^{D-2} \int_{\mathcal{D}} d^{2} \xi\left\langle\left(h_{i}\left(\xi^{1}, \xi^{2}\right)-h_{i}^{C M}\right)^{2 n}\right\rangle,
$$

one can retrieve the three-dimensional distribution of the colour field strength density $\rho(x, y, z)$ inside the flux tube. Indeed, choosing for simplicity the domain $\mathcal{D}$ sitting in the plane $z=0$, we can implicitly define such a density through the following equations

$$
w^{(2 n)}=\frac{\int \rho z^{2 n} d x d y d z}{\int \rho d x d y d z}
$$

As a consequence, the Fourier transform of $\rho$ can be written in terms of these moments as

$$
\tilde{\rho}(p)=\sum_{n=0}^{\infty}(-1)^{n} \frac{p^{2 n}}{(2 n) !} w^{(2 n)},
$$

where the integral of the density has been normalized to 1 . In the free gaussian model the above calculation can be performed explicitly using the Wick theorem, yielding simply

$$
w^{(2 n)}=(2 n-1) ! !\left(w^{2}\right)^{n}
$$

Inserting this result into Eq. (17) one finds that the density has a gaussian distribution

$$
\rho_{g}(z)=a \exp \left(-z^{2} / b^{2}\right)
$$

\subsection{Simulations on $\mathbb{Z}_{2}$ Gauge Model}

Though the logarithmic growth of the squared width of the flux tube is the most important and model-independent quantum effect predicted by the effective string description, it has 
not been observed until now, because it is an infrared phenomenon that can be seen only in very large Wilson loops, which are at the limit of the sizes reached by present numerical simulations on $S U(2)$ and $S U(3) 4 D$ gauge theories. However, it is nowadays possible to overcome this problem in the $\mathbb{Z}_{2} 3 D$ gauge model by exploiting some special features of this model.

The $3 D \mathbb{Z}_{2}$ gauge model on a cubic lattice is defined through the partition function

$$
Z_{\text {gauge }}(\beta)=\sum_{\left\{\sigma_{l}= \pm 1\right\}} \exp (-\beta S)
$$

where the action $S$ is a sum over all the plaquettes of the cubic lattice

$$
S=-\sum_{\square} \sigma_{\square} \quad, \quad \sigma_{\square}=\sigma_{l_{1}} \sigma_{l_{2}} \sigma_{l_{3}} \sigma_{l_{4}}
$$

This model can be translated into the usual $3 D$ Ising model by the usual Kramers-Wannier duality transformation

$$
\begin{aligned}
& Z_{\text {gauge }}(\beta) \propto Z_{\text {spin }}(\tilde{\beta}) \\
& \tilde{\beta}=-\frac{1}{2} \log [\tanh (\beta)]
\end{aligned}
$$

where $Z_{\text {spin }}$ is the partition function of the Ising model on the dual lattice

$$
Z_{\text {spin }}(\tilde{\beta})=\sum_{s_{i}= \pm 1} \exp (-\tilde{\beta} H(s))
$$

with

$$
H(s)=-\sum_{\langle i j\rangle} J_{\langle i j\rangle} s_{i} s_{j}
$$

where $i$ and $j$ denote nodes of the dual lattice and the sum is extended to the links $\langle i j\rangle$ connecting the nearest-neighbour sites. For the moment the couplings $J_{\langle i j\rangle}$ are all chosen equal to +1 .

Using the duality transformation it is possible to build up a one-to-one mapping of physical observables of the gauge system onto the corresponding spin quantities. For instance, the vacuum expectation value of a Wilson loop $W(C)$ can be expressed in terms 
of spin variables as follows. First, choose an arbitrary surface $\Sigma$ bounded by $C: \partial \Sigma=C$; then "frustrate" the links intersecting $\Sigma$, i.e. take $J_{\langle i j\rangle}=-1$ whenever $\langle i j\rangle \cap \Sigma \neq \emptyset$. Let us denote with $H^{\prime}(s)$ the Ising Hamiltonian with this choice of couplings: the new Ising partition function $Z_{\text {spin }}^{\prime}(\tilde{\beta})=\sum_{s_{i}= \pm 1} \exp \left(-\tilde{\beta} H^{\prime}(s)\right)$ describes a vacuum modified by the Wilson loop $W(C)$, which we shall call the $\mathrm{W}$-vacuum. A well-known consequence of duality is that

$$
\langle W(C=\partial \Sigma)\rangle_{\text {gauge }}=\frac{Z_{\text {spin }}^{\prime}}{Z_{\text {spin }}}=\left\langle\prod_{\langle i j\rangle \cap \Sigma \neq \emptyset} \exp \left(-2 \tilde{\beta} s_{i} s_{j}\right)\right\rangle_{\text {spin }}
$$

where the product is over all the dual links intersecting $\Sigma$.

One of the main difficulties in dealing with numerical simulations is that near a critical point the successive configurations generated by a Monte Carlo procedure are strongly correlated. Then it is hard to get a reliable set of thermalized configurations where the measurements can be done with sufficient accuracy. This phenomenon is known as critical slowing down.

A great advantage of mapping a gauge observable onto a spin observable is that a non-local cluster updating algorithm [12] can be used in place of the usual Metropolis or heat-bath methods. This kind of algorithm substantially reduces critical slowing down.

This allows us to probe the structure of the flux tube with a high accuracy. There is no analogous procedure for the other gauge theories.

The procedure is the following. A Wilson loop $W(C)$ is realized in the spin lattice by frustrating all the dual links intersecting a given surface $\Sigma$ bounded by $C$. These frustrated links modify the vacuum state so that the expectation value $\langle P\rangle_{W}$ of the plaquette, or better its spin counterpart described in Eq. (26), becomes a function of its relative position with respect to $W(C)$ and is related to the expectation value in the ordinary vacuum by

$$
\langle P\rangle_{W}=\langle W(C) P\rangle /\langle W(C)\rangle
$$

The difference between the expectation value of the plaquette in the vacuum modified by the presence of $W(C)$ and in the ordinary vacuum can be considered as a measure of 
the colour flux density of the flux tube. Choosing for instance as a probe a plaquette $P_{\|}$ parallel to the plane of the Wilson loop we can take

$$
\rho_{\|}(x, y, z)=\left\langle P_{\|}\right\rangle_{W}-\langle P\rangle
$$

Other orientations of the plaquette give approximately the same distribution (this is not the case for other systems [3, 目, 司]).

It is important to stress that within our procedure we do not need to evaluate directly the correlation function $\langle W(C) P\rangle$ between the plaquette and the Wilson loop, which is very difficult in ordinary lattice gauge model simulations and must be handled with special techniques like the cooling [3] or the smearing methods [四. Actually we only have to evaluate, according to Eq. (28), the mean expectation value of the plaquette with two different actions: the ordinary action and the one modified by the Wilson loop. This allows us to estimate $\rho$ with high accuracy even with relatively modest computational means. A preliminary account of these numerical simulations has been reported in Ref. [13].

Tab. II

\begin{tabular}{cccccc}
\hline$\beta$ & lattice size & $R$ & $T$ & \# data & $w_{o}^{2}$ \\
\hline 0.7516 & $24 \times 24 \times 48$ & 12 & 12 & 12500 & $19.0(1.1)$ \\
0.7460 & $22 \times 22 \times 40$ & 11 & 11 & 35000 & $12.3(5)$ \\
0.7460 & $30 \times 30 \times 48$ & 15 & 15 & 7000 & $15.6(8)$ \\
0.7460 & $60 \times 60 \times 48$ & 30 & 30 & 5000 & $20.3(4)$ \\
0.7460 & $120 \times 120 \times 32$ & 60 & 60 & 1000 & $24.8(6)$ \\
0.6543 & $64 \times 64 \times 32$ & 32 & 32 & 7400 & $2.57(2)$ \\
0.6543 & $124 \times 124 \times 24$ & 32 & 64 & 2000 & $2.79(5)$ \\
0.6543 & $96 \times 192 \times 32$ & 32 & 96 & 1700 & $2.75(5)$ \\
0.6543 & $124 \times 124 \times 20$ & 64 & 64 & 1000 & $2.95(3)$ \\
\hline
\end{tabular}

The first two columns show the $\beta$-values and the corresponding size of the lattices used in the present study, in the notation $L_{x} \times L_{y} \times L_{z}$ where $R$ and $T$ point in the $x$ and $y$ directions respectively. In the last four columns the size of the Wilson loops, the number of data and the corresponding estimates of the mean squared width of the flux tube are reported. 
We would also like to point out that the plaquette expectation values entering into Eq. (28), namely $\left\langle P_{\|}\right\rangle_{W}$ (with the $\mathrm{W}$-vacuum) and $\langle P\rangle$ (with the ordinary vacuum), are evaluated in distinct runs, so that the corresponding measurements are completely uncorrelated.

We analyzed three distinct values of the coupling $\beta$ and different sizes and shapes of square and rectangular Wilson loops, as can be seen from Tab. II.

For each run with a $\mathrm{W}$-vacuum, the Wilson loop is located in the $z_{0}$ plane. Crosscorrelations in the measurements of the plaquette expectation values defined at different distances $\left|z-z_{0}\right|$ from the Wilson loop have to be carefully considered in this procedure. To keep them under control we did five update sweeps of the lattice between two measurements, and we measured only plaquettes in a fixed $z-z_{0}$ slice each time. This means, for instance, that two measurements of the same observable were separated by $5 \times L_{z}$ sweeps, and there were at least 5 sweeps between measurements of different observables. From Tab. II one can see that each run contained between 100000 and 240000 sweeps.

Moreover, if one takes a plane parallel to the Wilson loop and looks at the shape of $\rho$ as a function of the $x-y$ coordinates on this plane, one can see that $\rho$ is approximately constant, except in a region extending over a few lattice spacings from the border of the Wilson loop [14]. Thus, in order to have a sufficiently stable evaluation of the width at the center of the Wilson loop, we averaged the plaquette expectation values $\left\langle P_{\|}\right\rangle_{W}$ over the $x-y$ directions, excluding a border along the Wilson loop perimeter of size four lattice spacings across.

A typical shape of the distribution $\rho$ measured on the symmetry axis of the loop as a function of the distance $z-z_{0}$ from the loop plane is drawn in Fig. 2. The dotted straight line corresponds to the plaquette expectation value in the ordinary vacuum, $\langle P\rangle=$ 0.8945 (1) for $\beta=0.7460$ (while we obtained $\langle P\rangle=0.70852(2)$ at $\beta=0.6543$ and $\langle P\rangle=$ $0.9108(2)$ at $\beta=0.7516)$ : it does not show appreciable finite size effects for the lattices we have considered. The other two curves will be explained in the last section. 
The mean squared width of the flux tube can be defined as

$$
w_{o}^{2}=\frac{\int z^{2} \rho_{\|} d x d y d z}{\int \rho_{\|} d x d y d z} .
$$

The integration region in the horizontal directions is taken over the central plateau where $\rho$ is approximately constant as a function of $x$ and $y$. The evaluation of the integral was performed numerically and all the errors were evaluated with the binning procedure and the jackknife method.

It is important to stress that the numerical integrations are not reliable if the asymptotic values of $\rho$ and $\langle P\rangle$ do not agree within errors. To this end it is crucial to take large lattice sizes compared to the Wilson loops (see Tab. II).

An important feature of Eq. (12) is that it can be written in a universal, $\beta$-independent form by expressing all the dimensional quantities $w_{o}, R$ and $R_{c}$ in units of $\sqrt{\sigma}$. Accordingly, we report in Fig. 3 the data of Tab. II and also very accurate data 15 for the mean squared width of fluid interfaces in the Ising model (black dots) for one of the couplings analyzed in our approach $(\beta=0.6543$ of the gauge model). Note indeed that, according to Tab. I, the squared width of the flux tube with periodic boundary conditions (which is the case of the fluid interfaces) is almost the same of that with fixed b.c.

The straight line represents a fit of all the data to the two-parameter formula

$$
\sigma w_{o}^{2}=a \log \sqrt{\sigma} R+b
$$

We used the values $\sigma=0.0107(1)$ at $\beta=0.7516$ and $\sigma=0.0189(1)$ at $\beta=0.7460$ according to Ref. [11]. Far from the scaling region, the string tension should be replaced by the stiffness $\kappa$. This is the case for $\beta=0.6543$ where we used $\kappa=0.234(9)$ according to Ref. [15]. One gets $b=0.173 \pm 0.009$ and $a=0.150 \pm 0.005$ in good agreement with the theoretical value $a_{t h}$ of the parameter $a$, fixed by Eq. (12) to be $a_{t h}=\frac{1}{2 \pi} \simeq 0.159155$.

Within high statistical accuracy these data clearly support a logarithmic widening of the flux tube in a range of quark separation $R$ over more than two orders of magnitude, starting at about $\sqrt{\sigma} R_{\min } \simeq 1.2$. 
The physical adimensional quantity $\sqrt{\sigma} R_{c}$ is expressed in terms of $a$ and $b$ as $\sqrt{\sigma} R_{c}=$ $\mathrm{e}^{-b / a}$. Using the fitted values of the parameters we get $\sqrt{\sigma} R_{c}=0.32 \pm 0.02$.

In order to compare these results with analogous data for other gauge groups, we may, with an abuse of language, express $\sqrt{\sigma}$ in the physical units of the QCD string. Then the infrared logarithmic behaviour observed in Fig. 3 starts at least at $R_{\min } \simeq 0.6 \mathrm{fm}$ while the maximal probed elongation of the flux tube corresponds to more than $100 \mathrm{fm}$. The data on $4 D S U(2)$ flux tubes [3, 四] cover now a distance up to $2 \mathrm{fm}$, but are still affected by strong systematic errors and are compatible also with a constant width for distances larger than $1 \mathrm{fm}$.

\section{Discussion}

The observed logarithmic widening of the flux tube exhibits, for the first time, a macroscopic effect due to the string-like motion of the colour flux tube. At present it is visible only in the $3 D \mathbb{Z}_{2}$ gauge system because only there it is possible to reach, using scaling and the duality property of this system, large separations of the quark sources. Nevertheless we expect a similar behaviour in all $3 D$ and $4 D$ gauge systems, because the appearance of string-like vibration modes of the colour flux tube is a universal property of the rough phase of whatever gauge model.

The agreement of the logarithmic growth of the mean squared width of the flux tube observed in $\mathbb{Z}_{2}$ gauge model with the predictions of the gaussian model seems rather impressive: indeed the universal slope of the logarithmic growth has been checked in a range (more than two order of magnitude) which is unusually wide in numerical simulations.

Admittedly, this prediction is not specific to the gaussian model: such a model is a member of a one-parameter family of conformal field theories, namely those with conformal anomaly $c=1$. These theories have the same local behaviour (hence produce the logarithmic growth of the mean squared width) but have different topological excitations described by winding modes around a circle of radius $r$. This is the parameter labelling 
the different members of the family and $r \rightarrow \infty$ corresponds to the gaussian limit.

Also the agreement with some predictions which are more specific of the gaussian model is rather good. In particular, the scale $R_{c}$ of the logarithmic growth depends also on the shape $t=T / R$ of the rectangular Wilson loop. As a consequence, the difference $\Delta w^{2}(R)=w_{t}^{2}(R)-w_{p}^{2}(R)$ between the mean squared width of the flux tube generated by the rectangular Wilson loop and that of the reference torus does not depend on $R$ but is a calculable function of the shape of $t$ as shown in Fig. 1. For fixed boundary conditions (dashed line) it reaches the asymptotic value of the infinite strip for very large values of $t$. The data extracted from numerical simulations in $\mathbb{Z}_{2}$ gauge model (black dots) seem to agree with this behaviour, even if the errors are too large to reach a definite conclusion. The fact that the data seem a bit lower than the expected values is probably due to a (non universal) finite size effect; indeed in the $2 D X Y$ model with fixed b.c. such an effect can be easily observed.

Another specific prediction of the gaussian model is that the scales $R_{c}$ for fixed and periodic boundary conditions are almost the same. This fact is nicely confirmed with high accuracy by Fig.3.

The only feature of the gaussian model which seems to slightly deviate form the observed data concerns the distribution of the flux density as a function of the distance from the Wilson loop. It should be, according to Eq. (19), a gaussian $\rho_{g}(z)$. Although $\rho_{g}$ (dashed line in Fig. 2) reproduces the raw features of the data, the fit is not very good. A much better fit is obtained by putting $\rho=\rho_{g} /\left(1-\gamma \rho_{g}\right)$, where $\gamma$ is another free parameter (continuous line). This is suggested by the assumption that the flux tube does actually self-interact and the interaction should be a power series in $\rho$.

In conclusion, the above results seem to indicate that the free gaussian model is a good approximation of the string-like behaviour of the flux tube at least in the infrared limit. 


\section{Acknowledgements}

We would like to thank M. Hasenbusch and K. Pinn for providing us with a very efficient non-local algorithm for the $2 D X Y$ model. This work has been partly supported by the Ministero dell'Università e della Ricerca Scientifica.

\section{Appendix}

The Green function of the $2 D$ free massless theory on a rectangle of size $R \times T$ with periodic boundary conditions on both sides, as given in Eq. (13), can be expanded as 17]

$$
\mathcal{G}(z)=-\frac{1}{2 \pi} \Re e\left\{\log z-\frac{(\Im m z)^{2}}{2 R T}-\sum_{k=1}^{\infty} G_{k} \frac{z^{2 k}}{2 k}\right\}
$$

with

$$
G_{k}=\frac{2 \zeta(2 k)}{R^{2 k}} E_{2 k}(i t) \quad, \quad t=T / R
$$

where $\zeta$ is the Riemann zeta function and the Eisenstein series have the following expansion

$$
E_{2 k}(\tau)=1+(-1)^{k} \frac{4 k}{B_{k}} \sum_{n=1}^{\infty} \sigma_{2 k-1}(n) q^{n} \quad, \quad q=\mathrm{e}^{2 i \pi \tau}
$$

$\sigma_{k}(n)$ denotes the sum of $k$ th powers of the positive divisors of $n$ and $B_{k}$ are the Bernoulli numbers.

Since $\mathcal{G}\left(z, z^{\prime}\right)$ is translationally invariant under $z \rightarrow z+a ; z^{\prime} \rightarrow z^{\prime}+a$, it is only a

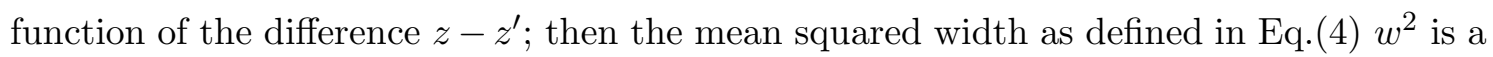
constant with respect to $z$ that we denote with $w_{p e r}^{2}(R)$. Performing the integrations we get at once

$$
\begin{aligned}
2 \pi \sigma w_{p e r}^{2}(R) & =\quad \log \left(R \sqrt{1+t^{2}} / 2 \varepsilon\right)+\frac{1}{2 t} \arctan t+\frac{t}{2} \arctan \frac{1}{t}-\frac{3}{2}-\frac{\pi t}{12} \\
& -\sum_{k=1}^{\infty} \frac{\left(1+t^{2}\right) E_{2 k}(i t)}{2 k t(2 k+2) !}\left(\left(1+t^{2}\right) \pi^{2}\right)^{k} B_{k} \sin ((2 k+2) \arctan t)
\end{aligned}
$$

This expansion converges only for $\sqrt{1+t^{2}}<2$ because there is a pole at $z=2 i \pi$ in the complex plane of the variable $z=\pi(R+i T) / R$. However it is possible to perform an 
analytic continuation which allows us to evaluate $w^{2}$ for any value of $t$, by resumming the potentially divergent part of the series. Indeed exploiting the well-known expansion

$$
\frac{z}{\mathrm{e}^{z}-1}=1-\frac{z}{2}-\sum_{k=1}^{\infty}(-1)^{k} \frac{B_{k}}{2 k !} z^{2 k}
$$

we can easily derive the result

$$
\sum_{k=1}^{\infty}(-1)^{k} \frac{B_{k}}{(2 k+2) !} \frac{z^{2 k+2}}{2 k}=\sum_{n=1}^{\infty} \frac{\mathrm{e}^{-n z}-1}{n^{3}}+\frac{z^{2}}{2} \log z+\frac{\pi^{2}}{6} z-\frac{3}{4} z^{2}-\frac{z^{3}}{12},
$$

which is useful for large $|z|$. Its insertion in Eq. (A.4) gives the wanted resummation, once one realizes that $E_{2 k}(i t)=1+O\left(\mathrm{e}^{-2 \pi t}\right)$. In particular, for large values of $t$ one gets the asymptotic expansion

$$
2 \pi \sigma w_{p e r}^{2}(R) \rightarrow \frac{\pi}{6} t+\log \frac{R}{2 \pi} \quad, \quad(t \rightarrow \infty)
$$

which is the expected behaviour in the one-dimensional limit.

For the rectangle with fixed boundary conditions one can use the same procedure, but in this case, owing to the fixed boundary, the Green function $G_{R \times T}$, is no longer translationally invariant. For the $\varepsilon$-dependent part of the Green function it is advantageous to use the following representation

$$
G_{R \times T}\left(z, z^{\prime}\right)=\mathcal{G}\left(z-z^{\prime}\right)+\mathcal{G}\left(z+z^{\prime}\right)-\mathcal{G}\left(z-\overline{z^{\prime}}\right)-\mathcal{G}\left(z+\overline{z^{\prime}}\right)
$$

where $\mathcal{G}$ is the periodic Green function for a torus of size $2 R \times 2 T$. Denoting with $w_{\text {fix }}^{2}(R)$ the mean squared width evaluated at the symmetry point of the Wilson loop we have

$$
\begin{array}{rc}
2 \pi \sigma w_{\text {fix }}^{2}(R)= & \log \left(R t / \sqrt{1+t^{2}} \varepsilon\right) \\
-\sum_{k=2}^{\infty} & \frac{\pi^{2 k} E_{2 k}(i t)}{2 k(2 k) !} B_{k}\left[\left(1+t^{2}\right)^{k} \cos (2 k \arctan t)-\left(-t^{2}\right)^{k}-1\right] \\
& +\chi(t)-\sum_{k=2}^{\infty} \frac{3 \pi^{k} E_{2 k}(i t)}{k t(2 k+2) !} B_{k} c_{k} \\
- & \frac{2 \pi}{R^{2} T^{2}} \int_{R \times T} \mathrm{~d}^{2} z \int_{R \times T} \mathrm{~d}^{2} z^{\prime} G_{R \times T}\left(z, z^{\prime}\right),
\end{array}
$$

with

$$
c_{k}=\quad 3\left(3^{2 k}+1\right)\left(1+t^{2}\right)^{k+1} \sin (2 k+2) \arctan t-
$$




$$
\begin{array}{cc}
\chi(t)= & \left(1+9 t^{2}\right)^{k+1} \sin (2 k+2) \arctan 3 t-\left(9+t^{2}\right)^{k+1} \sin (2 k+2) \arctan t / 3(\text { A. } 10) \\
f(x, y)= & {[9 f(R, T)-3 f(3 R, T)-3 f(R, 3 T)+f(3 R, 3 T)] / R T} \\
\frac{x y}{4} \log \left(x^{2}+y^{2}\right)+\frac{x^{2}}{4} \arctan \frac{y}{x}+\frac{y^{2}}{4} \arctan \frac{x}{y}
\end{array}
$$

Again the infinite sums can be resummed using Eq. (A.5).

The quadruple integral in Eq. (A.9) has the following representation

$$
\begin{array}{cc}
\frac{2 \pi}{R^{2} T^{2}} \int_{R \times T} \mathrm{~d}^{2} z \int_{R \times T} \mathrm{~d}^{2} z^{\prime} G_{R \times T}\left(z, z^{\prime}\right)= & \frac{\pi}{6 t}+\frac{31}{\pi^{4} t^{2}} \zeta(5) \\
& -\frac{64}{\pi^{4} t^{2}} \sum_{n=1}^{\infty} \frac{(2 n-1)^{-5}}{1+q^{2 n-1}}
\end{array}
$$

where $\zeta$ is the Riemann zeta function and $q=\exp (-\pi t)$.

A check of these formulas can be obtained by evaluating the $t \rightarrow \infty$ limit of Eq. (A.9), giving

$$
\lim _{t \rightarrow \infty} 2 \pi \sigma w_{f i x}^{2}(R)=\log (2 R / \pi \varepsilon)
$$

which coincides with the result for the infinite strip obtained by directly using the Green function of Tab. I.

\section{References}

[1] G. Parisi, Phys. Rev. D11 (1975) 970

G. 'tHooft, EPS International Conference, Palermo (1975)

S. Mandelstam, Phys. Rep. 23 C (1976) 245

[2] R. Sommer, Nucl. Phys. B291 (1987) 673

A. Di Giacomo, M. Maggiore and S. Olesjnik, Nucl. Phys. B347 (1990) 441

R.W. Haymaker and J. Wosiek, Phys. Rev. D43 (1991) 2676

R.W. Haymaker and Y. Peng, Phys. Rev. D47 (1993) 5104

[3] L. Del Debbio, A. Di Giacomo and Yu. Simonov, preprint IFUP-TH 11/94 (1994) 
[4] G.S. Bali, C. Schlichter and K. Schilling, preprint CERN-TH.7413/94 (1994)

[5] H.D Trottier and R.M. Woloshyn, Phys. Rev. D48 (1993) 2290.

[6] H.B. Nielsen and P. Olesen, Nucl. Phys. B61 (1973) 45

G.'t Hooft, Nucl. Phys. B72 (1974) 461

[7] M. Lüscher, K. Symanzik and P. Weisz, Nucl. Phys. B173 (1980) 365

M. Lüscher, Nucl. Phys. B180 [FS2] (1981) 317

[8] M. Lüscher, G. Münster and P. Weisz,Nucl. Phys. B180 [FS2] (1980) 1

[9] J.M. Kosterlitz and D.J. Thouless, J. Phys. C6 (1973) 1181

J.M. Kosterlitz, J. Phys. C7 (1974) 1046.

[10] P. Provero and S. Vinti, Physica A211 (1994) 436

[11] M. Caselle, R. Fiore, F. Gliozzi M. Hasenbusch, K. Pinn and S. Vinti, Nucl. Phys. B432 [FS] (1994) 590

[12] R.H. Swendsen and J.S. Wang, Phys. Rev. Lett. 58 (1987) 86

[13] M. Caselle, F. Gliozzi, U. Magnea and S. Vinti, Nucl. Phys. B42 (Proc. Suppl) (1995) 222

[14] F. Gliozzi, Proc. of the International Conference on Quark confinements and the hadron spectrum, Como (1994) 26

[15] M. Hasenbusch and K. Pinn, Physica A192 (1993) 342

[16] H.G. Evertz, M. Hasenbusch, M. Marcu, K. Pinn and S. Solomon, Phys. Lett. B254 $(1990) 185$

[17] L.V. Ahlfors, Complex Analysis, Mc Graw-Hill (1979), pag. 273 


\section{Figure Captions}

Fig. 1. Variation of the mean squared width of the flux tube as a function of the ratio $T / R$ calculated in the gaussian model and compared with numerical simulations in the $2 D X Y$ model (open circles and triangles for periodic and fixed boundary conditions respectively) and the $3 D \mathbb{Z}_{2}$ gauge theory (black dots). The continuous and dashed lines correspond to periodic and fixed boundary conditions, respectively. The dotted line indicates the infinite strip limit.

Fig. 2. Density of the flux tube generated by a $30 \times 30$ Wilson loop at $\beta=0.7460$. The dashed line is the fit to the gaussian distribution of Eq. (19). The continuous line is a fit to the function described in the text while the dotted line is the plaquette expectation value in the ordinary vacuum.

Fig. 3. Squared width of the flux tube in units of sigma. The open symbols are the data of the $\mathbb{Z}_{2}$ gauge theory and are taken from Tab. II, while the black circles are data from the Ising interface taken from Ref. [15]. 
fig. 1

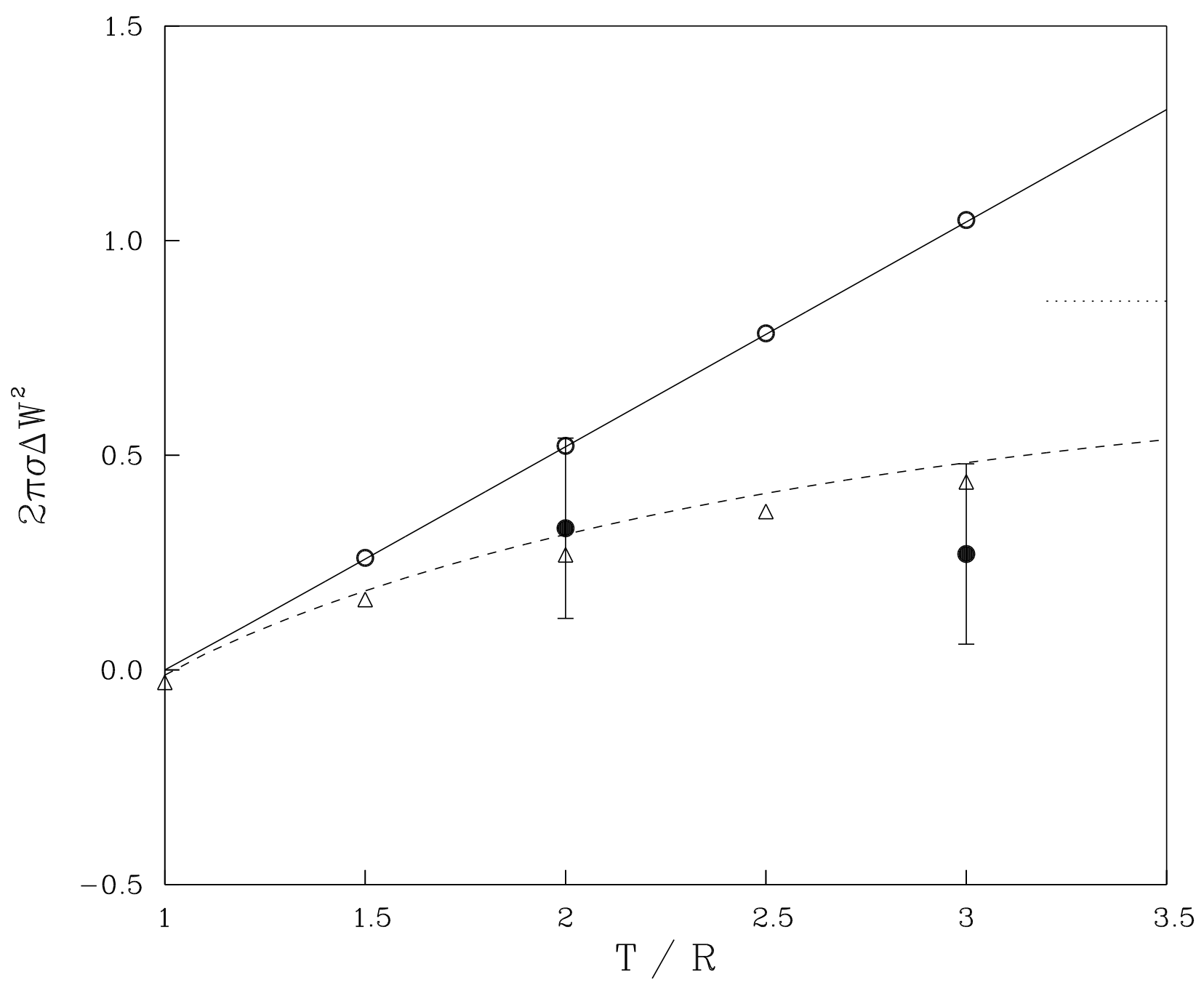


fig. 2

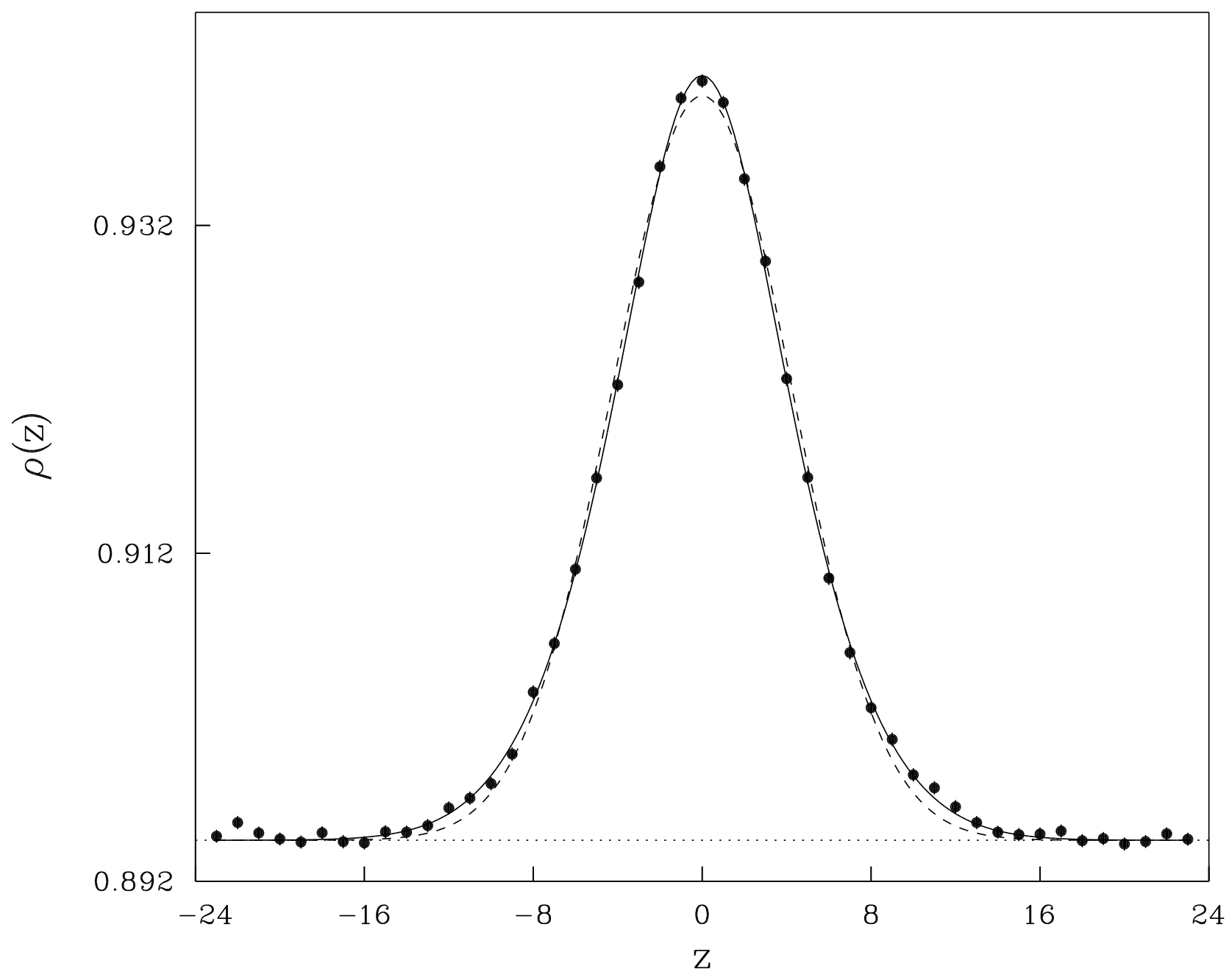


fig. 3

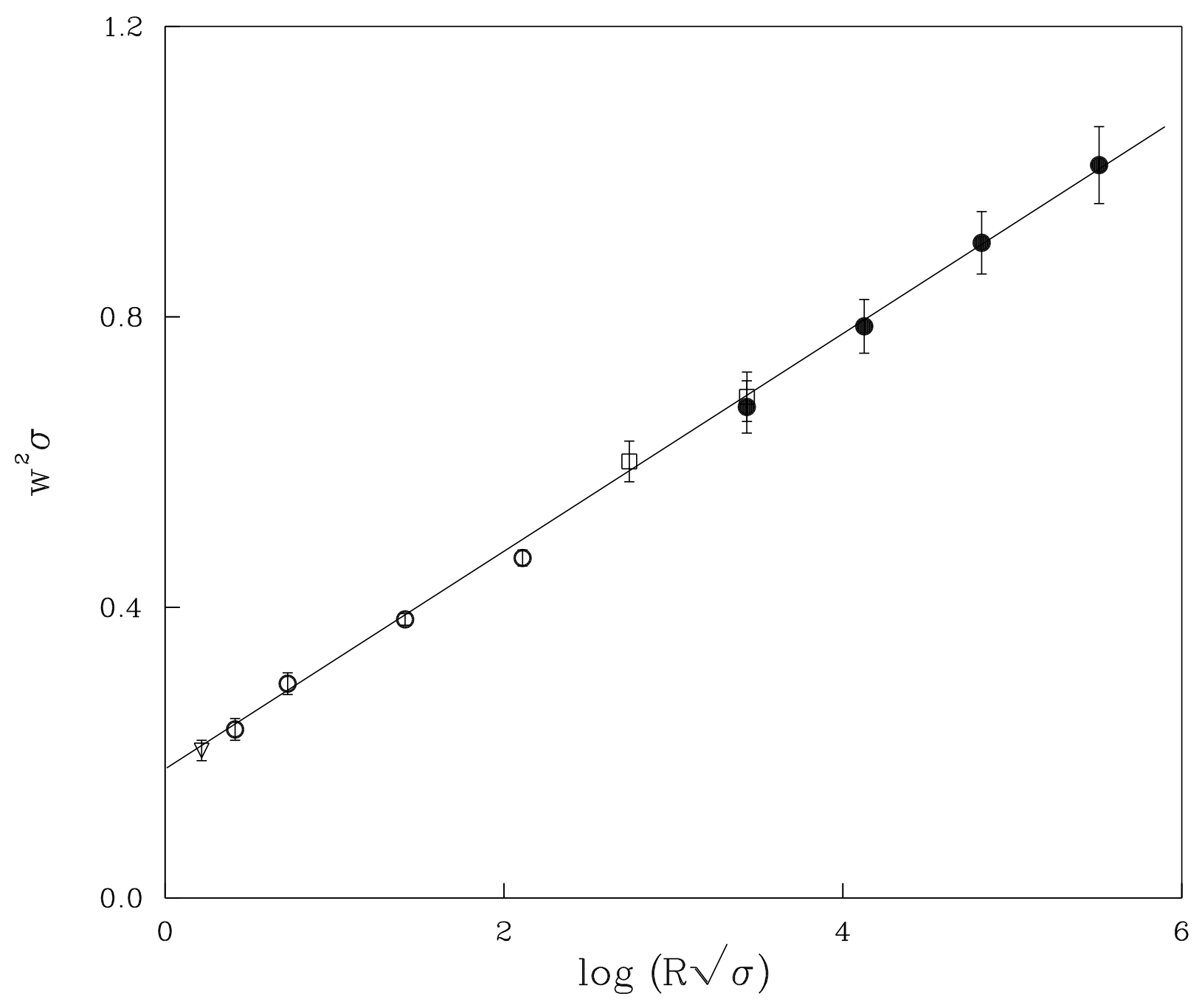

